\title{
HYPERSURFACES OF A FINSLER SPACE
}

\author{
HANNO RUND
}

Introduction. Certain aspects of the theory of subspaces of a Finsler space had been treated by the present author in earlier papers (7). These developments were based on an approach essentially different from the classical theory of Cartan (2) and subsequent writers, whose use of the element of support enables one to introduce the so-called "euclidean connection," which effects the vanishing of the covariant derivative of the metric tensor. A comprehensive treatment of the theory of subspaces of a Finsler space based on Cartan's point of view was given by Davies ${ }^{1}$ (4). However, the present writer seeks to dispense with the notion of element of support in the theory of Finsler spaces; in fact, a Finsler space is regarded as being locally Minkowskian instead of locally euclidean. From this point of view it is no longer possible to establish a euclidean connection in the above sense. This leads to a peculiar new geometrical picture; for instance, we have to deal with a set of normals attached to a point of a hypersurface instead of a single unique normal, nor are the covariant derivatives of these vectors tangential to the hypersurface. Two distinct differential forms play the rôle of the second fundamental form, while the number of principal directions at a point cannot be specified in the usual manner, due to lack of linearity.

The purpose of the present paper is to provide an analytical background and an extension of the results of (7), which is mainly geometrical in character. Some of the theorems of (7) will be derived once more in the course of our analysis: this is unavoidable, but it will be found that these results appear in a much improved form leading to new and more comprehensive theorems, of which the most interesting ones appear to be the distinct forms of the generalizations of the equations of Gauss and Codazzi of classical differential geometry. For the sake of geometrical clarity we shall deal with hypersurfaces instead of subspaces of arbitrary dimensions. Also, we shall briefly define the basic concepts concerning the theory of Finsler spaces, so that the present paper may be read independently.

We consider ${ }^{2}$ a space $F_{n}$ endowed with a local coordinate system

$$
x^{i}(i, j, \ldots=1,2, \ldots, n) .
$$

The distance between neighbouring points $x^{i}$ and $x^{i}+d x^{i}$ is defined by $d s=F\left(x^{h}, d x^{h}\right)$, where we make the following assumptions about the function $F$ :

Received August 26, 1955. This work was undertaken during the 1955 session of the Summer Research Institute at Queen's University, Kingston. The writer wishes to express his sincere gratitude to the Canadian Mathematical Congress.

${ }^{1}$ The reader is referred also to this paper as regards the relevant literature.

${ }^{2}$ For these and the following definitions see $(8, \S 2)$. 
(a) $F$ is of class $C^{4}$ in its $2 n$ arguments;

(b) $F$ is positive provided not all $d x^{h}=0$;

(c) $F$ is positively homogeneous of first degree in the $d x^{h}$;

(d) the form $g_{i j}\left(x^{h}, d x^{h}\right) \xi^{i} \xi^{j}>0$ for all $\xi^{i} \neq 0$ with any given argument $d x^{h}$, where we have put

$$
g_{i j}\left(x, x^{\prime}\right)=\frac{1}{2} \frac{\partial^{2} F^{2}\left(x, x^{\prime}\right)}{\partial x^{\prime i} \partial x^{\prime j}}, \quad x^{\prime i}=\frac{d x^{i}}{d s} .
$$

The quantities (0.1) are regarded as the components of the metric tensor of $F_{n}$; in view of hypothesis (c) the $g_{i j}$ are homogeneous of degree zero in the $x^{\prime i}$. Thus we have the useful identities:

$$
\frac{\partial g_{i j}\left(x, x^{\prime}\right)}{\partial x^{\prime k}} x^{\prime k}=\frac{\partial g_{i k}\left(x, x^{\prime}\right)}{\partial x^{\prime j}} x^{\prime k}=0 .
$$

The covariant differential of a vector-field $X^{i}\left(x^{k}\right)$ of $F_{n}$ is defined by

$$
D X^{i}=d X^{i}+P_{h k}^{i}(x, d x) X^{h} d x^{k},
$$

where

$$
P_{h k}^{i}\left(x, x^{\prime}\right) \equiv\left\{\begin{array}{c}
i \\
h k
\end{array}\right\}_{\left(x, x^{\prime}\right)}-\frac{1}{2} g^{i m}\left(x, x^{\prime}\right) \frac{\partial g_{h m}\left(x, x^{\prime}\right)}{\partial x^{\prime l}}\left\{\begin{array}{c}
l \\
j k
\end{array}\right\}_{\left(x, x^{\prime}\right)} x^{\prime j}
$$

On the other hand the covariant derivative of $X^{i}$ with respect to $x^{k}$ is given by

$$
X_{, k}^{i}=\frac{\partial X^{i}}{\partial x^{k}}+P_{h k}^{*_{i}} X^{h},
$$

where

$$
\text { (0.6) } P_{i j, h}^{*} \equiv g_{h k} P_{i j}^{*_{k}} \equiv[i j, h]-\frac{1}{2}\left(\frac{\partial g_{h j}}{\partial x^{\prime l}} P_{i k}^{l}+\frac{\partial g_{h i}}{\partial x^{\prime l}} P_{j k}^{l}-\frac{\partial g_{i j}}{\partial x^{\prime l}} P_{h k}^{l}\right) x^{\prime k} \text {. }
$$

We note that the $P_{h k}^{*_{i}}$ are symmetrical in $h$ and $k$, while for the $P_{h k}^{i}$ this is not true. $^{3}$ Owing to $(0.2)$ the following identities, which we shall have to use frequently in the sequel, may be shown to hold:

$$
\begin{gathered}
P_{h k}^{*_{i}}\left(x, x^{\prime}\right) x^{\prime k}=P_{h k}^{i}\left(x, x^{\prime}\right) x^{\prime k} ; \quad P_{h k}^{i}\left(x, x^{\prime}\right) x^{\prime h}=\left\{\begin{array}{c}
i \\
h k
\end{array}\right\}_{\left(x, x^{\prime}\right)} x^{\prime h} \\
P_{h k}^{*_{i}}\left(x, x^{\prime}\right) x^{\prime h} x^{\prime k}=\left\{\begin{array}{c}
i \\
h k
\end{array}\right\}_{\left(x, x^{\prime}\right)} x^{\prime h} x^{\prime k} .
\end{gathered}
$$

We may remark that for covariant differentiation along an arbitrary curve the covariant derivatives of the metric tensor do not in general vanish.

${ }^{3} \mathrm{E}$. T. Davies pointed out that the $P_{h k}^{*_{i}}$ are, in fact, identical to the $\Gamma_{h k}^{*_{i}}$ of Cartan (2). However, since we do not use the element of support, our covariant derivative still differs from that of Cartan. For instance, Ricci's Lemma holds in Cartan's theory, while this is not the case for the locally Minkowskian theory. 
1. The projection factors. Consider a hypersurface $F_{n-1}$ of $F_{n}$, defined by the equations

$$
x^{i}=x^{i}\left(u^{\alpha}\right),
$$

(throughout this paper Greek indices run from 1 to $n-1$; Latin indices from 1 to $n$ ) such that the matrix

$$
\left\|X_{\alpha}^{i}\right\|
$$

with

$$
X_{\alpha}^{i}=\frac{\partial x^{i}}{\partial u^{\alpha}}
$$

is of rank $n-1$. In general we have to consider a set of unit vectors ${ }^{4}$ normal to $F_{n-1}$ at a given point $P$ of $F_{n-1}$. These are defined firstly by the solutions $n^{i}$ of the equations

$$
n_{i} X_{\alpha}^{i} \equiv g_{i j}(x, n) n^{j} X_{\alpha}^{i}=0 .
$$

These solutions are normalized by means of the relation

$$
F(x, n)=1 \text { or } g_{i j}(x, n) n^{i} n^{j}=1 .
$$

The second set is defined by the solutions $n^{* i}$ of the equations

$$
g_{i j}\left(x, x^{\prime}\right) n^{*_{i}} X_{\alpha}^{j}=0,
$$

where $x^{\prime}$ is an arbitrary direction tangential to $F_{n-1}$ at $P$. Clearly the $n^{* i}$ are functions of this direction: $n^{* i}=n^{* i}\left(x, x^{\prime}\right)$. To each direction $x^{\prime}$ tangent to $F_{n-1}$ at $P$ corresponds such a vector $n^{* i}$; the totality of these vectors at $P$ defines a cone of directions, which we call the normal cone. Again we suppose the $n^{* i}$ to be normalized by means of the relations

$$
F\left(x, n^{*}\left(x, x^{\prime}\right)\right)=1 \quad \text { or } \quad g_{i j}\left(x, n^{*}\left(x, x^{\prime}\right)\right) n^{* i}\left(x, x^{\prime}\right) n^{* j}\left(x, x^{\prime}\right)=1 .
$$

For the sake of brevity we shall write

$$
n_{i}^{*}\left(x, x^{\prime}\right)=g_{i j}\left(x, x^{\prime}\right) n^{*_{j}}\left(x, x^{\prime}\right),
$$

where it is to be noted that this does not represent the covariant components of $n^{* i}$. We shall also have occasion to use the function defined by

$$
\psi\left(x, x^{\prime}\right)=g_{i j}\left(x, x^{\prime}\right) n^{* i}\left(x, x^{\prime}\right) n^{* j}\left(x, x^{\prime}\right) .
$$

From equations (1.3), (1.5), (1.7) and (1.8) we deduce that

$$
n_{i}^{*}\left(x, x^{\prime}\right)=\psi\left(x, x^{\prime}\right)\left[\cos \left(n, n^{*}\right)\right]^{-1} \cdot n_{i}
$$

where the Minkowskian cosine is defined (6, p. 62) on the indicatrix $F\left(x^{h}, \xi^{h}\right)=1$ of the Minkowskian tangent space to $F_{n}$ at $P$. The metric tensor of $F_{n-1}$ is given as usual by

$$
g_{\alpha \beta}\left(u, u^{\prime}\right)=g_{i j}\left(x, x^{\prime}\right) X_{\alpha}^{i} X_{\beta}^{j},
$$

where the directional argument $u^{\prime \alpha}$ tangent to $F_{n-1}$ satisfies

$$
x^{\prime i}=X_{\alpha}^{i} u^{\prime \alpha} \text {. }
$$

${ }^{4}$ For details concerning these definitions see (7, Part I, §4). 
Similarly we may also define a tensor independent of direction by putting

$$
\gamma_{\alpha \beta}(u)=g_{i j}(x, n) X_{\alpha}^{i} X_{\beta}^{j} .
$$

Corresponding to (1.10) and (1.12) we have to define two sets of inverse projection parameters, respectively dependent and independent of direction:

$$
\begin{aligned}
X_{i}^{\alpha}\left(x, x^{\prime}\right) & =g_{i j}\left(x, x^{\prime}\right) g^{\alpha \beta}\left(u, u^{\prime}\right) X_{\beta}^{j} ; \\
Y_{i}^{\alpha}(x) & =g_{i j}(x, n) \gamma^{\alpha \beta}(u) X_{\beta}^{j} .
\end{aligned}
$$

It follows that

$$
n^{*_{i}}\left(x, x^{\prime}\right) X_{i}^{\alpha}\left(x, x^{\prime}\right)=0 ; \quad n^{i} Y_{i}^{\alpha}=0 ;
$$

while

$$
X_{i}^{\alpha}\left(x, x^{\prime}\right) X_{\beta}^{i}=\delta_{\beta}^{\alpha} ; \quad Y_{i}^{\alpha} X_{\beta}^{i}=\delta_{\beta}^{\alpha} .
$$

For an arbitrary direction $x^{\prime}$ tangent to $F_{n-1}$ at $P$ we may decompose the metric tensor as follows:

$$
\begin{array}{r}
g_{i j}\left(x, x^{\prime}\right)=g_{\alpha \beta}\left(u, u^{\prime}\right) X_{i}^{\alpha} X_{j}^{\beta}+m_{\alpha}\left(u, u^{\prime}\right) X_{i}^{\alpha} n_{j}^{*}+n_{\alpha}\left(u, u^{\prime}\right) X_{j}^{\alpha} n_{i}^{*} \\
+\chi\left(u, u^{\prime}\right) n_{i}^{*} n_{j}^{*} .
\end{array}
$$

On multiplying this equation successively by $n^{* i}, X_{\beta}{ }^{j}$, it follows from the preceding identities that

$$
g_{i j}\left(x, x^{\prime}\right)=g_{\alpha \beta}\left(u, u_{)}^{\prime}\right) X_{i}^{\alpha}\left(x, x^{\prime}\right) X_{j}^{\beta}\left(x, x^{\prime}\right)+\frac{1}{\psi} n_{i}^{*}\left(x, x^{\prime}\right) n_{j}^{*}\left(x, x^{\prime}\right)
$$

Similarly

$$
g_{i j}(x, n)=\gamma_{\alpha \beta}(u) Y_{i}^{\alpha} Y_{j}^{\beta}+n_{i} n_{j} .
$$

On multiplying (1.17) and (1.18) by $g^{i k}\left(x, x^{\prime}\right)$ and $g^{i k}(x, n)$ respectively, we find that

$$
X_{\beta}^{k}(x) X_{j}^{\beta}\left(x, x^{\prime}\right)=\delta_{j}^{k}-\frac{1}{\psi} n^{*_{k}}\left(x, x^{\prime}\right) n_{j}^{*}\left(x, x^{\prime}\right),
$$

and

$$
X_{\beta}^{k}(x) Y_{j}^{\beta}(x)=\delta_{j}^{k}-n^{k} n_{j} .
$$

From these two equations together with (1.13) and (1.14) it follows that

$$
\begin{gathered}
g^{\alpha \beta}\left(u, u^{\prime}\right) X_{\alpha}^{i}(x) X_{\beta}^{j}(x)=g^{i j}\left(x, x^{\prime}\right)-\frac{1}{\psi} n^{*_{i}}\left(x, x^{\prime}\right) n^{*_{j}}\left(x, x^{\prime}\right), \\
\gamma^{\alpha \beta}(u) X_{\alpha}^{i} X_{\beta}^{j}=g^{i j}(x, n)-n^{i} n^{j} .
\end{gathered}
$$

Let

$$
X^{i}\left(x^{k}\right)=X_{\alpha}^{i} U^{\alpha}\left(u^{\beta}\right)
$$

be a continuous and continuously differentiable vector field tangent to $F_{n-1}$. The induced covariant derivative

$$
U_{, \gamma}^{\beta} \equiv \frac{\partial U^{\beta}}{\partial u^{\gamma}}+P_{\delta \gamma}^{*_{\beta}}\left(u, u^{\prime}\right) U^{\delta}
$$


of $U^{\beta}$ with respect to $F_{n-1}$ is defined by the projection onto $F_{n-1}$ of the covariant derivative $X_{, k}^{i}$ of $X^{i}$ with respect to $F_{n}$ :

$$
g_{i j}\left(x, x^{\prime}\right) X_{\gamma}^{j} X_{\alpha}^{k} X_{, k}^{i}=g_{\beta \gamma}\left(u, u^{\prime}\right) U_{, \alpha}^{\beta} .
$$

On substituting for the covariant derivative from (0.5) and differentiating (1.23) with respect to $u^{\alpha}$, a simple calculation yields

$$
\begin{aligned}
P_{\alpha \beta, \gamma}^{*}\left(u, u^{\prime}\right) \equiv g_{\delta \gamma}\left(u, u^{\prime}\right) P_{\alpha \beta}^{*_{\delta}}\left(u, u^{\prime}\right) & \\
& =g_{i j}\left(x, x^{\prime}\right) X_{\gamma}^{j}\left(\frac{\partial^{2} x^{i}}{\partial u^{\alpha} \partial u^{\beta}}+P_{h k}^{*_{i}}\left(x, x^{\prime}\right) X_{\alpha}^{h} X_{\beta}^{k}\right) .
\end{aligned}
$$

It is easily verified by means of (1.26) that under a transformation of the coordinates $u^{\alpha}$ of $F_{n-1}$, the quantities (1.24) form the components of a tensor in the sense indicated by their indices. Also, the $P_{\alpha \beta}^{\boldsymbol{*}_{\delta}}$ are symmetric in their lower indices. We remark that the induced connection coefficients need not necessarily be identical with the intrinsic coefficients of $F_{n-1}$, i.e. the connection coefficients which are derived from the $g_{\alpha \beta}$ and their derivatives in a manner analogous to that in which the $P_{h k}^{*_{i}}$ are derived from the $g_{i j}$ and their derivatives. ${ }^{5}$ However, if equation (1.10) is differentiated with respect to $u^{\gamma}$, one may obtain the transformation laws for the intrinsic Christoffel symbols $[\alpha \beta, \gamma]$ of the first kind (7, p. $369(3.5))$. On multiplying this equation with $u^{\prime \alpha} u^{\prime \beta}$, one finds in view of $(0.2)$ :

$$
[\alpha \beta, \gamma] u^{\prime \alpha} u^{\prime \beta}=g_{i j} X_{\gamma}^{j}\left(\frac{\partial^{2} x^{i}}{\partial u^{\alpha} \partial u^{\beta}}+\left\{\begin{array}{c}
i \\
h k
\end{array}\right\} X_{\alpha}^{h} X_{\beta}^{k}\right) u^{\prime \alpha} u^{\prime \beta} .
$$

Thus from (0.7), (1.11) and (1.26) it follows that

$$
[\alpha \beta, \gamma]_{\left(u, u^{\prime}\right)} u^{\prime \alpha} u^{\prime \beta}=P_{\alpha \beta, \gamma}^{*}\left(u, u^{\prime}\right) u^{\prime \alpha} u^{\prime \beta}
$$

analogously to $(0.7)$.

2. Normal curvature of the hypersurface. Let $C: x^{i}=x^{i}(s)$ be an arbitrary and continuously differentiable curve of $F_{n-1}$ passing through a given point $P\left(x^{i}\right)$ of $F_{n-1}$. The parameter $s$ is the arc-length. The unit tangent vector $d x^{i} / d s$ to $C$ at $P$ is denoted by $x^{\prime i}$, and throughout this section we shall suppose - unless otherwise stated - that the directional arguments of all subsequent functions are $x^{\prime i}$. At $P$ we have

$$
n_{i} x^{\prime i}=0 \text {. }
$$

In (7) we defined the normal curvature $R^{-1}\left(x, x^{\prime}\right)$ of $F_{n-1}$ for the direction $x^{\prime i}$ by putting

$$
R^{-1}\left(x, x^{\prime}\right)=n_{i} \frac{D x^{\prime i}}{D s}=-x^{i} \frac{D n_{i}}{D s}
$$

having arrived at this definition by considering variations of the unit normal in the neighbourhood of $P$. In the present section we shall derive a new

${ }^{5}$ This contradicts to some extent a statement made by the writer on p. 364 of (7). However, in view of (2.7) the results of (7) continue to hold. Compare also E. T. Davies (4). 
expression for (2.2), using a process entirely different from that of (7), our purpose being to find a more useful expression for the second fundamental form. Let us consider for the moment the special case for which the vector field $U^{\alpha}$ of equation (1.23) coincides with the tangent vectors $u^{\prime \alpha}$ of $C$. Using (1.23) and (1.27) we find

$$
\frac{\delta u^{\prime \alpha}}{\delta s} \equiv U_{, \gamma}^{\alpha} u^{\prime \gamma}=\frac{d u^{\prime \alpha}}{d s}+P_{\beta \gamma}^{*_{\alpha}} u^{\prime^{\beta}} u^{\prime \gamma}=\frac{d u^{\prime \alpha}}{d s}+\left\{\begin{array}{c}
\alpha \\
\beta \gamma
\end{array}\right\} u^{\prime \beta} u^{\prime \gamma},
$$

where $\delta$ denotes covariant differentiation in $F_{n-1}$. But on differentiating (1.2) along $C$ we have

$$
\frac{d x^{\prime i}}{d s}=\frac{\partial^{2} x^{i}}{\partial u^{\alpha} \partial u^{\beta}} u^{\prime \alpha} u^{\prime \beta}+X_{\alpha}^{i} \frac{d u^{\prime \alpha}}{d s} .
$$

On substituting (2.3) and (2.4) in the expression for $D x^{\prime i} / D s$ according to (0.3) we thus obtain (taking into account (0.7))

$$
\frac{D x^{\prime i}}{D s}=\frac{\partial^{2} x^{i}}{\partial u^{\alpha} \partial u^{\beta}} u^{\prime \alpha} u^{\prime \beta}+X_{\alpha}^{i} \frac{\delta u^{\prime \alpha}}{\delta s}-X_{\alpha}^{i} P_{\beta \gamma}^{*_{\alpha}} u^{\prime \beta} u^{\prime \gamma}+P_{h k}^{*_{i}} x^{\prime h} x^{\prime k},
$$

or, using (1.11),

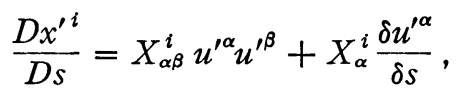

where we have put

$$
X_{\alpha \beta}^{i}=\frac{\partial^{2} x^{i}}{\partial u^{\alpha} \partial x^{\beta}}-X_{\gamma}^{i} P_{\alpha \beta}^{*_{\gamma}}+P_{h k}^{*_{i}} X_{\alpha}^{h} X_{\beta}^{k} .
$$

The expression (2.6) suggests that the $X_{\alpha \beta}^{i}$ may be regarded as generalised covariant derivatives of the $X_{\alpha}^{i}$ with respect to $u_{\beta}$ (as defined in (9), p. 124 for the case of a Riemannian space). This is indeed the case. Using the transformation properties of the connection coefficients, it can be shown by direct transformation that the $X_{\alpha \beta}^{i}$ have in fact the tensor properties as indicated by the position of their indices. We shall, however, omit this somewhat tedious calculation. Also, we note that they are symmetrical in their lower indices. On multiplying (2.5) by $n_{i}$ and taking into account (1.3), we find that the normal curvature (2.2) may be expressed in the form

$$
R^{-1}\left(x, x^{\prime}\right)=n_{i} X_{\alpha \beta}^{i} u^{\prime \alpha} u^{\prime \beta} .
$$

On multiplying (1.25) by $u^{\prime \alpha}$ it follows that the tangent vector $u^{\prime \alpha}$ to $C$ satisfies the relation

$$
g_{i j} X_{\gamma}^{j} \frac{D x^{\prime i}}{D s}=g_{\alpha \gamma} \frac{\delta u^{\prime \alpha}}{\delta s} .
$$

Now let us consider the geodesic $\Gamma$ of $F_{n-1}$ tangent to $C$ at $P$. A simple calculation shows that the Euler-Lagrange equations reduce to:

$$
\left(\frac{d u^{\prime \alpha}}{d s}\right)_{\Gamma}+\left\{\begin{array}{c}
\alpha \\
\beta \gamma
\end{array}\right\} u^{\prime \beta} u^{\prime \gamma}=0
$$


Hence it follows from $(2.3)$ that $\left(\delta u^{\prime \alpha} / \delta s\right)_{\Gamma}=0$, i.e. the geodesics are the autoparallel curves. Thus in view of $(2.8)$ the principal normal of $\Gamma$ (regarded as a curve of $F_{n}$ ) satisfies

$$
g_{i j}\left(x, x^{\prime}\right) X_{\gamma}^{j}\left(\frac{D x^{\prime i}}{D s}\right)_{\Gamma}=0 .
$$

Comparison with (1.5) shows that therefore

$$
\left(\frac{D x^{\prime i}}{D s}\right)_{\Gamma}=\frac{n^{*_{i}}}{\rho_{\Gamma}},
$$

where $\rho_{\Gamma}^{-1}$ is the curvature of $\Gamma$ (with respect to $F_{n}$ ) since $n^{* i}$ is a unit vector by (1.6). In contrast to the properties of hypersurfaces of locally euclidean spaces, $\rho_{\Gamma}^{-1}$ does not coincide with the normal curvature as defined by $(2.2)$; hence it is called the "secondary" normal curvature, denoted by $\left(R^{*}\left(x, x^{\prime}\right)\right)^{-1}$.

If we apply equation (2.5) (which holds for all curves of $F_{n-1}$ ) to the geodesic $\Gamma$, we have, in view of the remarks made above and (2.9):

$$
X_{\alpha \beta}^{i} u^{\prime \alpha} u^{\prime \beta}=n^{*_{i}} / R^{*}\left(x, x^{\prime}\right)
$$

and since this equation does not involve second derivatives it holds for all curves of $F_{n-1}$ tangent to $C$ at $P$. If we multiply $(2.10)$ by $n_{i}$, we have

$$
R^{-1}\left(x, x^{\prime}\right)=n_{i} n^{* i} / R^{*}\left(x, x^{\prime}\right),
$$

and hence

$$
R^{*}\left(x, x^{\prime}\right)=\cos \left(n, n^{*}\right) R\left(x, x^{\prime}\right),
$$

in agreement with $(7$, p. 200$)$ where this relation had been derived by a generalisation of Meusnier's theorem.

Furthermore, equation (2.10) suggests that the $X_{\alpha \beta}^{i}$ are normal to $F_{n-1}$. This is easily proved as follows. Using (1.11) and (1.10) we may write equation (1.25) in the form

$$
g_{i j} X_{\gamma}^{j} X_{\delta}^{i} P_{\alpha \beta}^{*_{\delta}}=g_{i j} X_{\gamma}^{j}\left(\frac{\partial^{2} x^{i}}{\partial u^{\alpha} \partial u^{\beta}}+P_{h k}^{*_{i}} X_{\alpha}^{h} X_{\beta}^{k}\right) \text {. }
$$

In view of (2.6) this becomes

$$
g_{i j}\left(x, x^{\prime}\right) \Lambda_{\gamma}^{j} X_{\alpha \beta}^{i}=0 .
$$

Comparing this with (1.5) we see that we may write

$$
X_{\alpha \beta}^{i}\left(u, u^{\prime}\right)=\Omega_{\alpha \beta}^{*}\left(u, u^{\prime}\right) n^{*_{i}}\left(x, x^{\prime}\right) .
$$

The $\Omega_{\alpha \beta}^{*}$ will be called the coefficients of the secondary second fundamental form, as distinct to an alternative, equally useful definition which we shall introduce presently. This nomenclature is justified by the fact that equations (2.10) and (2.13) together yield

$$
\left(R^{*}\left(x, x^{\prime}\right)\right)^{-1}=\Omega_{\alpha \beta}^{*}\left(u, u^{\prime}\right) u^{\prime \alpha} u^{\prime \beta}
$$

so that this fundamental form describes the secondary normal curvature, the $u^{\prime \alpha}$ being components of a unit vector since $s$ is the arc-length of $C$. 
If we multiply (2.13) by $n_{i}$ we have

$$
n_{i} X_{\alpha \beta}^{i}\left(u, u^{\prime}\right)=\Omega_{\alpha \beta}\left(u, u^{\prime}\right),
$$

where we have put

$$
\Omega_{\alpha \beta}\left(u, u^{\prime}\right)=\Omega_{\alpha \beta}^{*}\left(u, u^{\prime}\right) \cos \left(n, n^{*}\right) .
$$

We shall regard the $\Omega_{\alpha \beta}$ as the coefficients of the alternative second fundamental form. This equation is in agreement with the corresponding relation given in (7); we have to show, however, that the $\Omega_{\alpha \beta}$ as defined by (2.15) are identical to the $\Omega_{\alpha \beta}$ defined in (7) according to the relations

$$
\Omega_{\alpha \beta}=-\frac{1}{2}\left(n_{h, k}+n_{k, h}\right) X_{\alpha}^{h} X_{\beta}^{k} .
$$

Since the $P_{i k}^{*}$ are symmetric in $i$ and $k$, this definition reads:

$$
\Omega_{\alpha \beta}=-\frac{1}{2}\left(\frac{\partial n_{i}}{\partial u^{\beta}} X_{\alpha}^{i}+\frac{\partial n_{k}}{\partial u^{\alpha}} X_{\beta}^{k}\right)+n_{i} P_{h k}^{*_{i}} X_{\alpha}^{h} X_{\beta}^{k}
$$

But on differentiating (1.3) we have

$$
\frac{\partial n_{h}}{\partial u^{\beta}} X_{\alpha}^{h}=-n_{h} \frac{\partial^{2} x^{h}}{\partial u^{\alpha} \partial u^{\beta}},
$$

so that the above expression becomes

$$
\Omega_{\alpha \beta}=n_{i}\left(\frac{\partial^{2} x^{i}}{\partial u^{\alpha} \partial u^{\beta}}+P_{h k}^{*_{i}} X_{\alpha}^{h} X_{\beta}^{k}\right) .
$$

In view of (1.3) we may insert the additional term

$$
-X_{\delta}^{i} P_{\alpha \beta}^{*_{\delta}}
$$

into the bracket without changing the value of the right-hand side; thus the right-hand side of (2.17) becomes

$$
n_{i} X_{\alpha \beta}^{i}
$$

as a result of (2.6). Thus the definitions (2.15) and (2.17) are equivalent.

3. Principal directions. From (2.17) and (2.15) we deduce that the normal curvature of $F_{n-1}$ in the direction $d u^{\alpha}$ at $P$ is given by

$$
\left(R\left(x, x^{\prime}\right)\right)^{-1}=\frac{\Omega_{\alpha \beta}\left(u, u^{\prime}\right) d u^{\alpha} d u^{\beta}}{g_{\alpha \beta}\left(u, u^{\prime}\right) d u^{\alpha} d u^{\beta}} .
$$

The $(n-2)$-dimensional locus $\Omega_{\alpha \beta}\left(u, u^{\prime}\right) u^{\prime \alpha} u^{\prime \beta}=1$ in the hyperplane spanned by the $X_{\alpha}^{i}$ in the Minkowskian tangent space to $F_{n}$ at $P$ represents a generalization of the Dupin indicatrix. Principal directions are defined to be directions which are determined by those points on the Dupin indicatrix whose (Minkowskian) distance from the centre of the indicatrix assumes an extreme value relative to neighbouring points. In other words, principal directions are given by extreme values of $g_{\alpha \beta}\left(u, u^{\prime}\right) u^{\prime \alpha} u^{\prime \beta}$ subject to the condition $\Omega_{\alpha \beta}\left(u, u^{\prime}\right) u^{\prime \alpha} u^{\prime \beta}=1$, where $u^{\alpha}$ is being kept fixed. As a result of (3.1) principal directions are 
therefore directions for which the normal curvature assumes extreme values. According to the multiplier rule we therefore have to seek solutions of the equations ${ }^{6}$

$$
\frac{\partial}{\partial u^{\prime \gamma}}\left\{g_{\alpha \beta}\left(u, u^{\prime}\right) u^{\prime \alpha} u^{\prime \beta}+\lambda\left(\Omega_{\alpha \beta}\left(u, u^{\prime}\right) u^{\prime \alpha} u^{\prime \beta}-1\right)\right\}=0,
$$

or, in view of $(0.2)$,

$$
2 g_{\alpha \gamma}\left(u, u^{\prime}\right) u^{\prime \alpha}+2 \lambda \Omega_{\alpha \gamma}\left(u, u^{\prime}\right) u^{\prime \alpha}+\lambda \frac{\partial \Omega_{\alpha \beta}}{\partial u^{\prime \gamma}} u^{\prime \alpha} u^{\beta}=0 .
$$

This equation may be simplified considerably. We note first that

$$
\frac{\partial}{\partial x^{\prime \prime}}\left(\left\{\begin{array}{c}
i \\
h k
\end{array}\right\} x^{\prime h} x^{\prime k}\right)=\frac{\partial g}{\partial x^{\prime l}}[h k, m] x^{\prime h} x^{\prime k}+2 g^{i m}[l h, m] x^{\prime h}
$$

as a result of (0.2). Since $g^{i m} g_{i r}=\delta_{r}{ }^{m}$, this reduces to

$$
\begin{aligned}
\frac{\partial}{\partial x^{\prime \prime}}\left(\left\{\begin{array}{c}
i \\
h k
\end{array}\right\} x^{\prime h} x^{\prime k}\right) & \left.=2\left[\begin{array}{c}
i \\
l h
\end{array}\right\} x^{\prime h}-\frac{1}{2} g^{i m} \frac{\partial g_{i r}}{\partial x^{\prime l}}\left\{\begin{array}{c}
r \\
k h
\end{array}\right\} x^{\prime k} x^{\prime h}\right] \\
& =2 P_{l h}^{i} x^{\prime h}
\end{aligned}
$$

in virtue of (0.4) since $\partial g_{i r} / \partial x^{\prime l}$ is symmetric in all its indices (equation (0.1)). Now we differentiate $(0.7)$ with respect to $x^{\prime l}$; using (3.3) we obtain

$$
\frac{\partial P_{h k}^{*_{i}}}{\partial x^{\prime l}} x^{\prime h} x^{\prime k}+2 P^{*_{i}} x^{\prime k}=2 P_{l h}^{i} x^{\prime h} .
$$

On observing (0.7) once more we deduce immediately:

$$
\frac{\partial P_{h k}^{*_{i}}\left(x, x^{\prime}\right)}{\partial x^{\prime l}} x^{\prime h} x^{\prime k}=0 .
$$

But if we differentiate equation (2.18) with respect to $u^{\prime}$, we have, since $n_{i}$ is independent of direction,

$$
\frac{\partial \Omega_{\alpha \beta}}{\partial u^{\prime \gamma}}=n_{i} \frac{\partial P_{h k}^{*_{i}}}{\partial u^{\prime \gamma}} X_{\alpha}^{h} X_{\beta}^{k}
$$

and on multiplying this result by $u^{\prime \alpha} u^{\prime \beta}$ we may deduce that

$$
\frac{\partial \Omega_{\alpha \beta}}{\partial u^{\prime \gamma}} u^{\prime \alpha} u^{\prime \beta}=0
$$

having taken into account (1.11) and (3.4). Thus equation (3.2) reduces to

$$
g_{\alpha \gamma}\left(u, u^{\prime}\right) u^{\prime \alpha}=-\lambda \Omega_{\alpha \gamma}\left(u, u^{\prime}\right) u^{\prime \alpha} .
$$

Multiplying this result by $u^{\prime \gamma}$ it follows from (3.1) that $\lambda=-R$, so that the equation for principal directions finally reads:

$$
g_{\alpha \gamma}\left(u, u^{\prime}\right) u^{\prime \alpha}=R\left(u, u^{\prime}\right) \Omega_{\alpha \gamma}\left(u, u^{\prime}\right) u^{\prime \alpha},
$$

${ }^{6}$ In (7) principal directions were defined similarly, but for a second fundamental form whose coefficients are independent of direction. It is shown here that the method applies also to the general case. 
where $\left(R\left(u, u^{\prime}\right)\right)^{-1}$ is the normal curvature corresponding to a solution of (3.6).

Since this is not a linear eigenvalue problem, nothing can be said about the number of possible independent solutions. However, let us assume for the moment that at least two independent solutions

$$
u_{(1)}^{\prime \alpha}, u_{(2)}^{\prime \alpha}
$$

corresponding to two distinct normal curvatures $\left.R_{(1)}\right)^{-1}$ and $R_{(2)}{ }^{-1}$ exist, this assumption being geometrically feasible. Writing down the two equations (3.6) corresponding to each of these solutions and multiplying them by

$$
u_{(2)}^{\prime \gamma} \text { and } u_{(1)}^{\prime \gamma}
$$

respectively, we have

$$
g_{\alpha \gamma}\left(u, u_{(1)}^{\prime}\right) u_{(1)}^{\prime \alpha} u_{(2)}^{\prime \gamma}=R_{(1)} \Omega_{\alpha \gamma}\left(u, u_{(1)}^{\prime}\right) u_{(1)}^{\prime \alpha} u_{(2)}^{\prime \gamma},
$$

together with

$$
g_{\alpha \gamma}\left(u, u_{(2)}^{\prime}\right) u_{(2)}^{\prime \alpha} u_{(1)}^{\prime \gamma}=R_{(2)} \Omega_{\alpha \gamma}\left(u, u_{(2)}^{\prime}\right) u_{(2)}^{\prime \alpha} u_{(1)}^{\prime \gamma} .
$$

Since the $u^{\prime \alpha}$ are unit vectors, the left-hand sides of these equations are by definition the Minkowskian cosines

$$
\cos \left(u_{(1)}^{\prime}, u_{(2)}^{\prime}\right) \text { and } \cos \left(u_{(2)}^{\prime}, u_{(1)}^{\prime}\right)
$$

respectively (6). Thus on subtraction we find the following relations between principal directions:

$$
\frac{\cos \left(u_{(1)}^{\prime}, u_{(2)}^{\prime}\right)-\cos \left(u_{(2)}^{\prime}, u_{(1)}^{\prime}\right)}{R_{(1)} R_{(2)}}=\left[\frac{\Omega_{\alpha \gamma}\left(u, u_{(1)}^{\prime}\right)}{R_{(2)}}-\frac{\Omega_{\alpha \gamma}\left(u, u_{(2)}^{\prime}\right)}{R_{(1)}}\right] u_{(1)}^{\prime \alpha} u_{(2)}^{\prime \gamma}
$$

This is a generalisation of the orthogonality relations between principal directions of hypersurfaces of locally euclidean spaces. For if the cosine were symmetric in its directional arguments, and if the coefficients of the second fundamental form were independent of direction, it would follow from (3.8) that principal directions would correspond to conjugate directions of the Dupin indicatrix, and hence either $(3.7 \mathrm{a})$ or $(3.7 \mathrm{~b})$ would lead to the law of orthogonality.

4. The covariant derivative of the unit normals. For a large number of problems it is essential to have a convenient expression for the covariant derivative of the various unit normal vectors. In this section we shall obtain such formulae and use them in a discussion of a few simple applications. In the next section these relations will be essential in the derivation of the generalised Gauss-Codazzi equations. A difficulty peculiar to locally Minkowskian spaces is the fact that the covariant derivative of the unit normals is not tangential to the hypersurface: it is due to this fact alone that our formulae are more complicated than the corresponding relations in Riemannian geometry. 
We define the tensor

$$
n_{, \beta}^{i}=n_{, k}^{i} X_{\beta}^{k}=\frac{\partial n^{i}}{\partial u^{\beta}}+P_{n k}^{*_{i}} n^{h} X_{\beta}^{k}
$$

By writing

$$
C_{i j k}\left(x, x^{\prime}\right)=g_{i j, k}(x, n)
$$

for the covariant derivative of the $g_{i j}(x, n)$ with respect to $x^{k}(8, \S 2)$, we find that covariant differentiation of (1.3) with respect to $u^{\beta}$ gives

$$
\Omega_{\alpha \beta}=-C_{i j k} X_{\beta}^{k} X_{\alpha}^{i} n^{j}-g_{i j}(x, n) X_{\alpha}^{i} n_{, \beta}^{j} .
$$

Now let us decompose $n_{, \beta}^{j}$ as follows:

$$
n_{, \beta}^{j}=A_{\beta}^{\delta} X_{\delta}^{j}+\nu_{\beta} n^{j},
$$

where the coefficients are to be determined as follows. Multiplying (4.4) by

$$
g_{i j}(x, n) X_{\alpha}^{i}
$$

we find in virtue of (1.3), (1.12) and (4.3)

$$
\gamma_{\alpha \delta} A_{\beta}^{\delta}=-\Omega_{\alpha \beta}-C_{i j k} X_{\beta}^{k} X_{\alpha}^{i} n^{j} .
$$

Also, on multiplying (4.4) by $n_{j}$ we see that

$$
\nu_{\beta}=n_{j} n_{, \beta}^{j} \text {. }
$$

Differentiating (1.4) covariantly with respect to $u_{\beta}$ and taking into account (4.1) and (4.2) we have

$$
\nu_{\beta}=-\frac{1}{2} C_{i j k} n^{i} n^{j} X_{\beta}^{k}
$$

On substituting (4.5) and (4.7) in (4.4) we deduce that

$$
n_{, \beta}^{j}=-\gamma^{\alpha \delta} \Omega_{\alpha \beta} X_{\delta}^{j}-C_{i h k} n^{h} X_{\beta}^{k}\left[\gamma^{\alpha \delta} X_{\alpha}^{i} X_{\delta}^{j}+\frac{1}{2} n^{i} n^{j}\right] .
$$

Hence in view of (1.22) we have the desired formula:

$$
n_{, \beta}^{j}=-\gamma^{\alpha \delta} \Omega_{\alpha \beta} X_{\delta}^{j}-C_{i n k} n^{h} X_{\beta}^{k}\left[g^{i j}(x, n)-\frac{1}{2} n^{i} n^{j}\right] .
$$

At first sight one might be led to suspect that the term $C_{i n k} n^{n}$ implicitly involves the derivatives $\partial n^{l} / \partial u^{\beta}$ : this is not the case, however, since the term

$$
\frac{\partial g_{i h}(x, n)}{\partial x^{\prime l}} \cdot \frac{\partial n^{l}}{\partial x^{k}} X_{\beta}^{k} n^{h}
$$

containing these derivatives vanishes identically in view of (0.2). Thus the covariant derivative (4.8) depends only on positional coordinates and the direction $x^{\prime i}$ along which we are differentiating, i.e. it is the same for all curves of $F_{n-1}$ which have a common tangent $x^{\prime i}$ at the point under consideration.

It is also necessary to evaluate the covariant derivatives of the generators $n^{* i}$ of the normal cone. By a process similar to the one described above, we find after some calculation: 


$$
\begin{aligned}
n_{, \beta}^{*_{j}}= & -\psi\left(x, x^{\prime}\right) g^{\alpha \delta}\left(u, u^{\prime}\right) \Omega_{\alpha \beta}^{*} X_{\delta}^{j}+\frac{n^{*_{j}}\left(x, x^{\prime}\right)}{2 \psi\left(x, x^{\prime}\right)} \psi_{, \beta} \\
& -C_{i n k}^{*} n^{*_{h}}\left(x, x^{\prime}\right) X_{\beta}^{k}\left(g^{i j}\left(x, x^{\prime}\right)-\frac{n^{*_{i}}\left(x, x^{\prime}\right) n^{*_{j}}\left(x, x^{\prime}\right)}{2 \psi\left(x, x^{\prime}\right)}\right),
\end{aligned}
$$

where

$$
C_{i j k}^{*}\left(x, x^{\prime}\right)=g_{i j, k}\left(x, x^{\prime}\right) .
$$

In contrast to (4.8), equation (4.9) suffers from the drawback that the term $\psi_{, \beta}$ on the right-hand side involves the derivatives of the tangent $x^{\prime i}$ to the curve along which we are differentiating, so that (4.9) depends on the curve under consideration.

As a first application of these formulae let us consider principal directions as defined in the preceding section. From (4.1) and (4.8) we have

$$
\frac{D n^{j}}{D s}=-\gamma^{\alpha \delta} \Omega_{\alpha \beta} u^{\prime \beta} X_{\delta}^{j}-C_{i n k} n^{h} x^{\prime k}\left[g^{i j}(x, n)-\frac{1}{2} n^{i} n^{j}\right] .
$$

Using (4.2) we may write

$$
\frac{D n_{i}}{D s}=C_{i n k} n^{h} x^{\prime k}+g_{i j}(x, n) \frac{D n^{j}}{D s}
$$

and on substituting from (4.11) in the last term of this equation, we find after some simplification

$$
\frac{D n_{i}}{D s}=-g_{i j}(x, n) X_{\delta}^{j} \gamma^{\epsilon \delta} \Omega_{\epsilon \beta} u^{\prime \beta}+\frac{1}{2} n_{i}\left(C_{h j k} n^{h} n^{j} x^{\prime k}\right) .
$$

Hence from (1.3) and (1.12) we deduce in particular:

$$
X_{\alpha}^{i} \frac{D n_{i}}{D s}=-\Omega_{\alpha \beta} u^{\beta} .
$$

If $\left(R\left(x, x^{\prime}\right)\right)^{-1}$ is the normal curvature corresponding to a principal direction $x^{\prime i}$ of $F_{n-1}$, we have from (3.6) and (4.13):

$$
X_{\alpha}^{i} \frac{D n_{i}}{D s}=-\left(R\left(x, x^{\prime}\right)\right)^{-1} g_{\alpha \beta}\left(u, u^{\prime}\right) u^{\prime \beta},
$$

or, if we denote the covariant components of the unit vector representing the principal direction by $y_{\alpha}$,

$$
X_{\alpha}^{i} \frac{D n_{i}}{D s}=-\left(R\left(u, u^{\prime}\right)\right)^{-1} y_{\alpha} .
$$

Thus the projection of the covariant differential of the unit normal onto $F_{n-1}$ coincides with the principal direction. This is a generalisation of the classical formula of Rodrigues. ${ }^{7}$ It should be noted, however, that in contrast to the classical theory, the covariant differential of $n^{i}$ has a normal component, in general non-vanishing, even in the case of principal directions.

${ }^{7}$ In (7), Part II, a similar result was obtained for principal directions corresponding to the alternative second fundamental form. 
Another simple application of the equation (4.8) is the generalisation of an important formula due to Bianchi (1, p. 450) concerning the deformation of hypersurfaces in classical differential geometry, which was later generalised by Davies (3, p. 291) in his theory of the second and third fundamental forms of subspaces of a Riemannian space. At each point $x^{i}$ of $F_{n-1}$ we construct the unit normal $n^{i}$; the locus of points whose coordinates are $x^{i}+\epsilon n^{i}$ (where $\epsilon$ is an arbitrarily small quantity) form a new hypersurface $\bar{F}_{n-1}$. Let $P\left(x^{i}\right)$, $Q\left(x^{i}+d x^{i}\right)$ be two neighbouring points of $F_{n-1}$, a distance $d s$ apart. There will be two neighbouring points $P^{\prime}\left(x^{i}+\epsilon n^{i}\right), Q^{\prime}\left(x^{i}+d x^{i}+\epsilon\left(n^{i}+d n^{i}\right)\right)$ on $\bar{F}_{n-1}$ corresponding to $P, Q$ respectively, where $d n^{i}$ corresponds to the change in $n^{i}$ as we pass from $P$ to $Q$. If we denote the distance between $P^{\prime}$ and $Q^{\prime}$ by $d \bar{s}$, we have

$$
d \bar{s}^{2}=g_{i j}\left(x^{h}+\epsilon n^{h}, d x^{h}+\epsilon d n^{h}\right)\left(d x^{i}+\epsilon d n^{i}\right)\left(d x^{j}+\epsilon d n^{j}\right)
$$

Expanding the expression on the right-hand side and dividing by $d s$, we find

$$
\left(\frac{d \bar{s}}{d s}\right)^{2}-1=\epsilon\left[2 g_{i j}\left(x, x^{\prime}\right) x^{\prime i} \frac{d n^{j}}{d s}+\frac{\partial g_{i j}\left(x, x^{\prime}\right)}{\partial x^{k}} n^{k} x^{\prime j} x^{\prime i}\right]
$$

or, after some rearrangement,

$$
\left(\frac{d \bar{s}}{d s}\right)^{2}-1=2 \epsilon g_{i j}\left(x, x^{\prime}\right) x^{\prime i}\left[\frac{d n^{j}}{d s}+\left\{\begin{array}{c}
j \\
h k
\end{array}\right\}_{\left(x, x^{\prime}\right)} n^{h} x^{\prime k}\right],
$$

where we have neglected terms involving higher powers of $\epsilon$.

But from definition (0.4) we have

$g_{i j}\left(x, x^{\prime}\right) x^{\prime i} \frac{D n^{j}}{D s}=g_{i j}\left(x, x^{\prime}\right) x^{\prime i}\left[\frac{d n^{j}}{d s}+\left\{\begin{array}{c}j \\ h k\end{array}\right\} n^{h} x^{\prime k}-\frac{n_{h}}{2} g^{j m} \frac{\partial g_{h m}}{\partial x^{\prime l}}\left\{\begin{array}{c}l \\ p k\end{array}\right\} x^{\prime p} x^{\prime k}\right]$, and in view of the relation $g_{j m} g^{i j}=\delta_{m}^{i}$ it follows from (0.2) that the last term on the right-hand side vanishes. Thus (4.15) becomes

$$
\left(\frac{d \bar{s}}{d s}\right)^{2}-1=2 \epsilon g_{i j}\left(x, x^{\prime}\right) x^{i} \frac{D n^{j}}{D s} .
$$

In the locally euclidean case this would simply become the formula of Bianchi or Davies (loc. cit.) as a result of (2.2) and (3.1). In the present case the position is a little more complicated: using (4.11), (1.10) and (1.22), equation (4.16) may be written in the form

$$
\begin{gathered}
\left(\frac{d \bar{s}}{d s}\right)^{2}-1=-2 \epsilon\left\{g_{\beta \gamma}\left(u, u^{\prime}\right) \gamma^{\beta \epsilon} \Omega_{\epsilon \alpha} u^{\prime \gamma} u^{\prime \alpha}\right. \\
\left.+g_{r j}\left(x, x^{\prime}\right) X_{\gamma}^{r} C_{i h k} n^{h} X_{\alpha}^{k} u^{\prime \alpha} u^{\prime \gamma}\left[\gamma^{\epsilon \beta} X_{\epsilon}^{i} X_{\beta}^{j}+\frac{1}{2} n^{i} n^{j}\right]\right\} .
\end{gathered}
$$

Applying (1.10) once more together with (1.17), we find after some simplification

$$
\left(\frac{d \bar{s}}{d s}\right)^{2}-1=-2 \epsilon \omega_{\alpha \gamma}\left(u, u^{\prime}\right) u^{\prime \alpha} u^{\prime \gamma}
$$


where we have put

$$
\omega_{\alpha \gamma}\left(u, u^{\prime}\right)=g_{\beta \gamma}\left(u, u^{\prime}\right) \gamma^{\beta \epsilon}\left[\Omega_{\alpha \epsilon}+C_{i n k} n^{h} X_{\alpha}^{k}\left(X_{\epsilon}^{i}+\frac{1}{2} X_{j}^{\delta} \gamma_{\epsilon \delta} n^{i} n^{j}\right)\right] .
$$

It appears, therefore, that in the general case the $\Omega_{\alpha \gamma}$ do not possess all the essential properties which one may attribute to them in Riemannian geometry. Nevertheless, it would not be feasible to introduce the $\omega_{\alpha \gamma}$ of (4.18) as the coefficients of an alternative second fundamental form since these quantities are not symmetrical in their lower indices.

5. The equations of Gauss and Codazzi. In order to find the desired relations between the coefficients $\Omega_{\alpha \beta}$ of the second fundamental form of $F_{n-1}$ and the curvature tensor of $F_{n}$, it is necessary to express the $X_{\alpha \beta}^{i}$ in terms of the unit normal vector $n^{i}$ in a manner analogous to equation (2.13). We therefore define a new set of quantities $\omega_{\alpha \beta}^{i}$ by means of the equations

$$
X_{\alpha \beta}^{i}=\Omega_{\alpha \beta} n^{i}+\omega_{\alpha \beta}^{i} .
$$

It is simple to derive an explicit expression for the $\omega_{\alpha \beta}^{i}$. Using (2.13) and (2.16) we see that (5.1) may be written as

$$
\omega_{\alpha \beta}^{i}=\Omega_{\alpha \beta}\left\{n^{*_{i}} \sec \left(n, n^{*}\right)-n^{i}\right\} .
$$

If we decompose the vector $n^{i}$ in the form

$$
n^{i}=\phi n^{*_{i}}-\mu^{\alpha} X_{\alpha}^{i}
$$

we find on multiplication of this equation by $n_{i}$ that $\phi=\sec \left(n, n^{*}\right)$ in virtue of (1.3); and similarly, on multiplying (5.3) by $X_{i}^{\delta}$ and taking into account (1.15) and (1.16), we see that

$$
\mu^{\delta}=-n^{i} X_{i}^{\delta}
$$

The vector $\mu^{\delta}\left(u, u^{\prime}\right)$ thus expresses the difference between the unit normal vectors $n^{i}$ and $n^{* i}\left(x, x^{\prime}\right)$. From (5.3) and (5.4) we finally deduce that

$$
\omega_{\alpha \beta}^{i}=\Omega_{\alpha \beta} \mu^{\delta} X_{\delta}^{i}
$$

where $\mu^{\delta}$ is given by (5.4).

It may be verified by direct calculation that the process of generalised covariant differentiation ${ }^{8}$ leads to the identities

$$
X_{\alpha \beta \gamma}^{i}-X_{\alpha \gamma \beta}^{i}=R_{. \alpha \beta \gamma}^{\delta} X_{\delta}^{i}-R_{. h k l}^{i} X_{\alpha}^{h} X_{\beta}^{k} X_{\gamma}^{l},
$$

where the subscripts $\beta$ and $\gamma$ on the left-hand side indicate covariant differentiation with respect to $u^{\beta}$ and $u^{\gamma}$ respectively, and where $R_{. h k l}^{i}$ and $R_{. \alpha \beta \gamma}^{\delta}$ represent the curvature tensors (8, p. $91(3.7)$ ) of $F_{n}$ and $F_{n-1}$. From (5.6) and (5.1) we therefore have

${ }^{8}$ Throughout this section the directional arguments are the components of a vector $x^{\prime} i$ tangent to the hypersurface, corresponding to the direction along which the covariant differentiation takes place. 


$$
\begin{gathered}
X_{\delta}^{i} R_{. \alpha \beta \gamma}^{\delta}=R_{. h k l}^{i} X_{\alpha}^{h} X_{\beta}^{k} X_{\gamma}^{l}+n^{i}\left(\Omega_{\alpha \beta, \gamma}-\Omega_{\alpha \gamma, \beta}\right) \\
+\omega_{\alpha \beta \gamma}^{i}-\omega_{\alpha \gamma \beta}^{i}+\Omega_{\alpha \beta} n_{, \gamma}^{i}-\Omega_{\alpha \gamma} n_{, \beta}^{i}
\end{gathered}
$$

where $\omega_{\alpha \beta \gamma}^{i}$ denotes the generalised covariant derivative of $\omega_{\alpha \beta}^{i}$ with respect to $u^{\gamma}$.

In this equation we substitute the values of $n_{, \beta}^{i}$ and $n_{. \gamma}^{i}$ as given by equation (4.8). After some factorisation, equation (5.7) finally reduces to the form

$$
\begin{aligned}
& X_{\delta}^{i}\left[R_{. \alpha \beta \gamma}^{\delta}-\gamma^{\delta \epsilon}\left(\Omega_{\alpha \gamma} \Omega_{\epsilon \beta}-\Omega_{\alpha \beta} \Omega_{\epsilon \gamma}\right)\right]=R_{. h k l}^{i} X_{\alpha}^{h} X_{\beta}^{k} X_{\gamma}^{l} \\
& +\left(\omega_{\alpha \beta \gamma}^{i}-\omega_{\alpha \gamma \beta}^{i}\right)-g^{i h}(x, n) C_{h l k} n^{l}\left(\Omega_{\alpha \beta} X_{\gamma}^{k}-\Omega_{\alpha \gamma} X_{\beta}^{k}\right) \\
& -n^{i}\left[\Omega_{\alpha \gamma, \beta}-\Omega_{\alpha \beta, \gamma}-\frac{1}{2} C_{h l k} n^{h} n^{l}\left(\Omega_{\alpha \beta} X_{\gamma}^{k}-\Omega_{\alpha \gamma} X_{\beta}^{k}\right)\right] .
\end{aligned}
$$

We multiply this equation by $g_{i j}(x, n) X_{\lambda}^{j}$. In view of (1.12) and (1.3) we thus obtain

$$
\begin{gathered}
\gamma_{\delta \lambda} R_{. \alpha \beta \gamma}^{\delta}-\left(\Omega_{\alpha \gamma} \Omega_{\lambda \beta}-\Omega_{\alpha \beta} \Omega_{\lambda \gamma}\right) \\
=g_{i j}(x, n) R_{. h k l}^{i} X_{\alpha}^{h} X_{\beta}^{k} X_{\gamma}^{l} X_{\lambda}^{j}-C_{j l k} n^{l}\left(\Omega_{\alpha \beta} X_{\gamma}^{k}-\Omega_{\alpha \gamma} X_{\beta}^{k}\right) X_{\lambda}^{j} \\
\quad+g_{i j}(x, n) X_{\lambda}^{j}\left(\omega_{\alpha \beta \gamma}^{i}-\omega_{\alpha \gamma \beta}^{i}\right) .
\end{gathered}
$$

If, on the other hand, we multiply equation (5.8) by $g_{i j}(x, n) n^{j}$ we find, after taking into account (1.3) and (1.4) and suitable rearrangement of indices:

$$
\begin{aligned}
& g_{i j}(x, n) R_{. h k l}^{i} n^{j} X_{\alpha}^{h} X_{\beta}^{k} X_{\gamma}^{l}=\left(\Omega_{\alpha \gamma, \beta}-\Omega_{\alpha \beta, \gamma}\right) \\
& +\frac{1}{2} C_{j h k} n^{j} n^{h}\left(\Omega_{\alpha \beta} X_{\gamma}^{k}-\Omega_{\alpha \gamma} X_{\beta}^{k}\right)-g_{i j}(x, n) n^{j}\left(\omega_{\alpha \beta \gamma}^{i}-\omega_{\alpha \gamma \beta}^{i}\right) .
\end{aligned}
$$

Equations (5.9) and (5.10) represent the generalisations of the equations of Gauss and Codazzi of classical differential geometry. On comparing these equations with the corresponding equations (5, p. $162(4.11)$ and (4.12)) of Riemannian geometry, we see that the essential differences (apart from the impossibility of contracting terms with different directional arguments) lie in the additional terms involving the $C_{i j k}$ and the $\omega_{\alpha \beta \gamma}^{i}$. This, again, is owing to the fact that the covariant derivative of the unit normals is not tangential to the hypersurface and that different normals have to be taken into account. However, it is possible to remove the terms in (5.9) and (5.10) involving the $\omega_{\alpha \beta \gamma}^{i}$ and to replace these terms by expressions involving the $\Omega_{\alpha \beta}$. If we write down the generalised covariant derivative of (5.5) and use (5.1) we find

$$
\omega_{\alpha \beta \gamma}^{i}=\left(\Omega_{\alpha \beta, \gamma} \mu^{\delta}+\Omega_{\alpha \beta} \mu_{, \gamma}^{\delta}\right) X_{\delta}^{i}+\Omega_{\alpha \beta} \mu^{\delta}\left(\Omega_{\delta \gamma} n^{i}+\omega_{\delta \gamma}^{i}\right) .
$$

Hence, on observing (1.12) and (1.3), we thus obtain

$$
g_{i j}(x, n) X_{\lambda}^{j} \omega_{\alpha \beta \gamma}^{i}=\gamma_{\lambda \delta}\left(\Omega_{\alpha \beta, \gamma} \mu^{\delta}+\Omega_{\alpha \beta} \mu_{, \gamma}^{\delta}\right)+g_{i j}(x, n) X_{\lambda}^{j} \omega_{\delta \gamma}^{i} \Omega_{\alpha \beta} \mu^{\delta} .
$$

In the last term of this expression we substitute from (5.5), so that (1.12) may be applied once more. Thus the last equation becomes

$$
g_{i j}(x, n) X_{\lambda}^{j} \omega_{\alpha \beta \gamma}^{i}=\gamma_{\lambda \delta}\left(\Omega_{\alpha \beta, \gamma} \mu^{\delta}+\Omega_{\alpha \beta} \mu_{, \gamma}^{\delta}+\Omega_{\epsilon \gamma} \Omega_{\alpha \beta} \mu^{\epsilon} \mu^{\delta}\right) .
$$


Also, it follows from (5.5) and (1.3) that

$$
n_{i} \omega_{\alpha \beta}^{i}=0 .
$$

Hence on multiplying (5.11) by $n_{i}$ and taking into account (1.3) and (1.4), we find that

$$
n_{i} \omega_{\alpha \beta \gamma}^{i}=\Omega_{\alpha \beta} \Omega_{\delta \gamma} \mu^{\delta} .
$$

On substituting from (5.12) and (5.13) in (5.9) and (5.10) respectively, we obtain the equations of Gauss and Codazzi in their final form:

$$
\begin{aligned}
\gamma_{\delta \lambda} R_{\alpha \beta \gamma}^{\delta} & -\left(\Omega_{\alpha \gamma} \Omega_{\lambda \beta}-\Omega_{\alpha \beta} \Omega_{\lambda \gamma}\right)=g_{i j}(x, n) R_{. h k l}^{i} X_{\alpha}^{h} X_{\beta}^{k} X_{\gamma}^{l} X_{\lambda}^{j} \\
& -C_{j l k} n^{l}\left(\Omega_{\alpha \beta} X_{\gamma}^{k}-\Omega_{\alpha \gamma} X_{\beta}^{k}\right) X_{\lambda}^{j} \\
& +\gamma_{\lambda \delta l} \mu^{\delta}\left(\Omega_{\alpha \beta, \gamma}-\Omega_{\alpha \gamma, \beta}\right)+\left(\Omega_{\alpha \beta} \mu_{, \gamma}^{\delta}-\Omega_{\alpha \gamma} \mu_{, \beta}^{\delta}\right) \\
& \left.+\left(\Omega_{\epsilon \gamma} \Omega_{\alpha \beta}-\Omega_{\alpha \gamma} \Omega_{\epsilon \beta}\right) \mu^{\epsilon} \mu^{\delta}\right],
\end{aligned}
$$

together with

$$
\begin{aligned}
& g_{i j}(x, n) R_{. h k l}^{i} n^{j} X_{\alpha}^{h} X_{\beta}^{k} X_{\gamma}^{l}=\left(\Omega_{\alpha \gamma, \beta}-\Omega_{\alpha \beta, \gamma}\right) \\
& \quad+\frac{1}{2} C_{j h k} n^{j} n^{h}\left(\Omega_{\alpha \beta} X_{\gamma}^{k}-\Omega_{\alpha \gamma} X_{\beta}^{k}\right)-\left(\Omega_{\alpha \beta} \Omega_{\delta \gamma}-\Omega_{\alpha \gamma} \Omega_{\delta \beta}\right) \mu^{\delta} .
\end{aligned}
$$

It is clear that different forms of the Gauss-Codazzi equations are obtained when one considers the secondary second fundamental form

$$
\Omega_{\alpha \beta}^{*} u^{\prime \alpha} u^{\prime \beta}
$$

together with a given generator $n^{* i}\left(x, x^{\prime}\right)$ of the normal cone; i.e. when equation (2.13) is used instead of (2.15). The calculation proceeds along similar lines to the one outlined above, and will therefore be omitted. Instead of (5.7) one obtains

$$
\begin{aligned}
& g^{\delta \epsilon}\left(u, u^{\prime}\right) X_{\delta}^{i}\left[R_{\epsilon \alpha \beta \gamma}\left(u, u^{\prime}\right)-\psi\left(\Omega_{\alpha \gamma}^{*} \Omega_{\epsilon \beta}^{*}-\Omega_{\alpha \beta}^{*} \Omega_{\epsilon \gamma}^{*}\right)\right] \\
& =R_{h k l}^{i}\left(x, x^{\prime}\right) X_{\alpha}^{h} X_{\beta}^{k} X_{\gamma}^{l}-g^{i h}\left(x, x^{\prime}\right) C_{h l k}^{*} n^{*}{ }^{*}\left(\Omega_{\alpha \beta}^{*} X_{\gamma}^{k}-\Omega_{\alpha \gamma}^{*} X_{\beta}^{k}\right) \\
& -n^{* i}\left[\left(\Omega_{\alpha \gamma, \beta}^{*}-\Omega_{\alpha \beta, \gamma}^{*}\right)-\frac{1}{2 \psi} C_{l h k}^{*} n^{*_{h}} n^{{ }^{*} l}\left(\Omega_{\alpha \beta}^{*} X_{\gamma}^{k}-\Omega_{\alpha \gamma}^{*} X_{\beta}^{k}\right)\right. \\
& \left.-\frac{1}{2 \psi}\left(\Omega_{\alpha \beta}^{*} \psi, \gamma-\Omega_{\alpha \gamma}^{*} \psi, \beta\right)\right] .
\end{aligned}
$$

On multiplying this equation by $g_{i j}\left(x, x^{\prime}\right) X_{\lambda}{ }^{j}$ we obtain in virtue of (1.10) and (1.6):

$$
\begin{aligned}
& R_{\lambda \alpha \beta \gamma}\left(u, u^{\prime}\right)-\psi\left(\Omega_{\alpha \gamma}^{*} \Omega_{\lambda \beta}^{*}-\Omega_{\alpha \beta}^{*} \Omega_{\lambda \gamma}^{*}\right) \\
& \quad=R_{j h k l}\left(x, x^{\prime}\right) X_{\lambda}^{i} X_{\alpha}^{h} X_{\beta}^{k} X_{\gamma}^{l}-C_{j l k}^{*} n^{*} X_{\lambda}^{j}\left(\Omega_{\alpha \beta}^{*} X_{\gamma}^{k}-\Omega_{\alpha \gamma}^{*} X_{\beta}^{k}\right) .
\end{aligned}
$$

In analogy to (5.15) we find similarly by means of (1.6) and (1.8):

$$
\begin{aligned}
& R_{j h k l}\left(x, x^{\prime}\right) n^{{ }_{j}} X_{\alpha}^{h} X_{\beta}^{k} X_{\gamma}^{l}=\psi\left(\Omega_{\alpha \gamma, \beta}^{*}-\Omega_{\alpha \beta, \gamma}^{*}\right) \\
& \quad+\frac{1}{2} C_{j h k}^{*} n^{{ }_{h}} n^{* j}\left(\Omega_{\alpha \beta}^{*} X_{\gamma}^{k}-\Omega_{\alpha \gamma}^{*} X_{\beta}^{k}\right)-\frac{1}{2}\left(\Omega_{\alpha \beta}^{*} \psi_{, \gamma}-\Omega_{\alpha \gamma}^{*} \psi_{, \beta}\right) .
\end{aligned}
$$


Equations (5.17) and (5.18) thus represent alternative forms of the generalisations of the equations of Gauss and Codazzi. As regards the study of imbedding problems it is probably advantageous to base any such discussion on equations (5.14) and (5.15).

In conclusion we may remark that the equations of Gauss and Codazzi in Riemannian geometry are known to be dependent on each other to a certain extent: whether this is true also for the general case is still an open question.

\section{REFERENCES}

1. L. Bianchi, Lezioni di Geometria Differenziale, vol II, parte II (Bologna, 1924).

2. E. Cartan, Les espaces de Finsler, Actualités 79 (Paris, 1934).

3. E. T. Davies, On the second and third fundamental forms of a subspace, J. London Math. Soc. 12 (1937), 290-295.

4. Subspaces of a Finsler space. Proc. London Math. Soc. Ser. 2, 49 (1945), 19-39.

5. L. P. Eisenhart, Riemannian geometry (Princeton, 1926).

6. H. Rund, Differentialgeometrie der Minkowskischen Räume, Arch. d. Math. 3 (1952), 60-69.

7. The theory of subspaces of a Finsler space, Part I: Math. Z. 56 (1952), 363-375; Part II: Math. Z. 57 (1953), 193-210.

8. - On the analytical properties of curvature tensors in Finsler spaces, Math. Ann. 127 (1954), 82-104.

9. C. E. Weatherburn, Introduction to tensor calculus and Riemannian geometry (Cambridge, 1938).

University of Natal, South Africa 